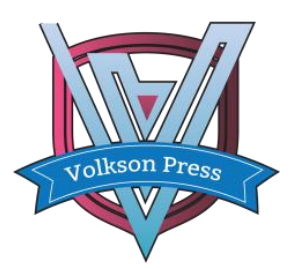

\title{
BRAIN ACTIVATION LOCALIZATION ALGORITHM BASED ON ALTERNATING VOLUME MAXIMIZATION METHODS
}

\author{
Liushengqian $^{{ }^{*}}$ Fengbao $^{2}$ Zhanglieping $^{1}$ and Sunxuan ${ }^{1}$ \\ ${ }^{1}$ Collage of mechanical and control engineering, Guilin University of Technology, NO12 Jiangan road, guilin, China. \\ ${ }^{2}$ Collage of electronic information and automation, Guilin College of aerospace industry, Jingji road, guilin, China. \\ *Corresponding Author Email: 117980887@qq.com
}

This is an open access article distributed under the Creative Commons Attribution License, which permits unrestricted use, distribution, and reproduction in any medium, provided the original work is properly cited

\section{ARTICLE DETAILS}

\section{Article History:}

Received 02 october 2017 Accepted 06 october 2017 Available online 11 november 2017

Keywords

Alternating Volume Maximization Methods, function Magnetic Resonance Imaging (fMRI), Independent Component Analysis, Convex Optimization, Voxel Selectio

\section{ABSTRACT}

Recent studies showed that the independence assumption for Independent components analysis (ICA) based method is sometime violated in practice for function Magnetic Resonance Imaging (fMRI) data analysis. In order to overcome this problem, we proposed a new blind separation method for brain image data analysis. Combined with the natural characteristics of fMRI data, the new method exploits sparsity and non-negativity of sources to blindly decompose fMRI data. The proposed method estimates the source components in a convex analysis framework. It is achieved in two steps. First, it shows that source components serve as extreme points of a convex set, which is constructed based on the observed fMRI data. Next, all the source components can be estimated by finding extreme points of the convex set obtained in the first step. For determination of extreme points, alternating volume maximization is exploited to obtain robustness of fMRI model errors to improve accuracy of blind separation accuracy of fMRI data. Numerical results showed that, compared with ICA based method, voxels selected by the proposed method are more related to task function. The proposed method has high quality solution and the solving efficiency and can reliably process the brain image data.

\section{Introduction}

Brain science research is one of the hotspot and frontier of scientific research at home and abroad, it belongs to the interdisciplinary, content involves brain science, biology, medicine, mathematics, automatic control, sensor, computer science, information science, materials science, and many other fields [1,2]. Functional Magnetic Resonance ImagingIs one of the commonly used technology for brain research, mainly by the neural activity of blood flow or metabolic changes in the signal, through image processing and imaging methods, the activity of the brain in the form of visual images expressed. FMRI technology provides a way to study brain science without trauma, living detection, radioactivity, high spatial resolution, and multiple repetitions $[3,4]$.

Due to the particularity of fMRI technology, the imaging data has the characteristics of high data dimension, low SNR, and a large number of unknown patterns of brain activity, leading to the traditional data-driven method, Such as Principle Component Analysis Independent Component Analysis, It is difficult to achieve the desired effect $[5,6]$.

Principal component analysis (PCA) method is the basic idea of observations of fMRI data between each other through linear transformation into unrelated component, and select one of the biggest variance components as the most relevant and experiment task. There is an implicit assumption in this process that the task information is contained in the largest component of the variance. This hypothesis is the premise of: tasks related to neural response signal energy enjoys an absolute advantage relative to the background noise, to think that the biggest variance component contains all the information in neural response signal. In practical application, in order to improve the signal-tonoise ratio, a certain experiment is repeated several times and the data collected is suppressed and the additive noise is suppressed [7].

The basic assumption of the independent component analysis (ICA) method is that the brain activation mode caused by the task is independent in the time domain, airspace or time and space joint domain. However, Friston and others believe that the assumption of independence is too strict for fMRI data. Due to the phenomenon of "functional integration", a brain region may participate in multiple stimulation response processes simultaneously, which can overlap the spatial distribution of the brain activation area of different source components. At this point, the independent hypothesis is contrary to the actual medical condition. The common feature of PCA and ICA data driving methods is that the algorithm performance depends fundamentally on the rationality and accuracy of the prior hypothesis of fMRI data [8].

Based on the convex optimization and the general characteristics of the fMRI data, this paper puts forward the localization algorithm of brain activation region using the source component sparse and non-negative. Compared to the independence assumption, the sparse and non-negative assumptions are more consistent with the characteristics of fMRI data Since the entire cerebral cortex contains billions of body elements, and the body element associated with the task is only a small part of the brain, the space is sparse and distributed in the brain, showing the sparsity [9-11]. In addition, the fMRI data is essentially a grayscale image that records brain activity, and the gray value of the image is nonnegative , So the nonnegative hypothesis is also reasonable [12].

Nonnegative linear weighted sum signal source component, and USES the convex optimization techniques will signal blind source separation problem into a series of convex optimization problem, and on the premise of give up independence assumption fMRI signal separation, in the search for data related to the experiment of the signal components and more the useful signal contained in the data model.

In the process of signal separation, using the fMRI data obtained from the observation of building closed convex set, and transforms the estimation problem of the source component to determine the problem of convex sets the endpoint, in the process of determining convex set the endpoint, using the method of Alternating Volume Maximization to construct the optimal approximation convex set, To improve the accuracy of convex set endpoint estimation [13]. Compared with the linear programming method, the AVM method has good robustness to the error of fMRI model. In addition, the Task Related component of the fMRI signal separation (Consistent Task Related, CTR) can be further used in the study of brain functional area localization (also known as somatoin selection). The experimental results 
also show that the proposed method can be used to decompose the fMRI data and the localization analysis based on CTR component can also obtain reliable results.

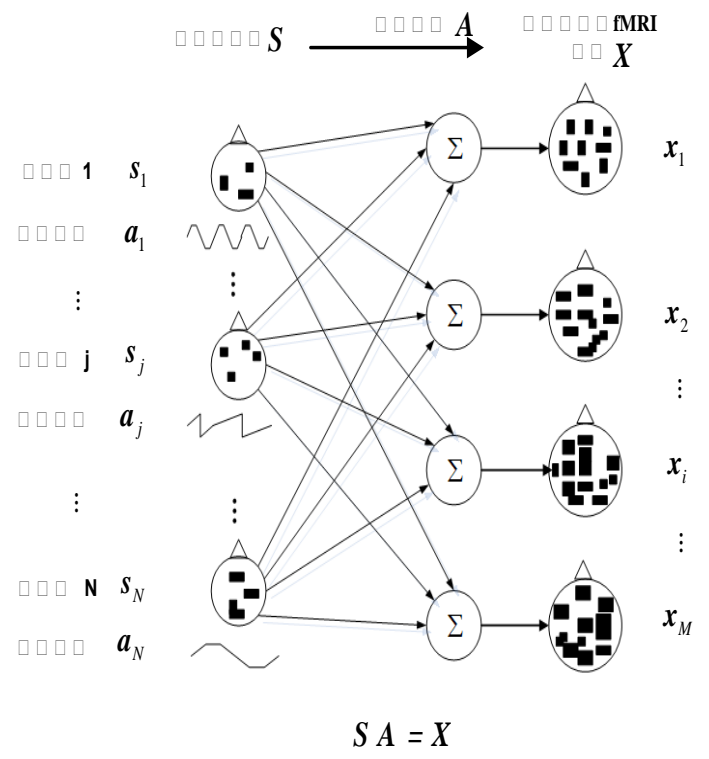

Figure 1: fMRI of signal modeling

As shown in figure 1, the fMRI data obtained at different moments is observed $\boldsymbol{X}=\left[\boldsymbol{x}_{1}, \cdots, \boldsymbol{x}_{i}, \cdots, \boldsymbol{x}_{M}\right] \in R^{L \times M} \quad$ It's a different source $S=\left[s_{1}, \cdots, s_{j}, \cdots, s_{N}\right] \in R^{L \times N} \quad$ Linear mixing ( The different neuropsychological processes of different source components indicate the spatial pattern of different brain signal sources ) , The signal model can be expressed as

$$
X=S A
$$

Among them, $\boldsymbol{A}=\left[\boldsymbol{a}_{1}, \ldots, \boldsymbol{a}_{j}, \ldots, \boldsymbol{a}_{N}\right]^{T} \in R^{N \times M}$

It's a hybrid matrix,$\quad \boldsymbol{a}_{j}=\left[a_{j}[1], \ldots, a_{j}[M]\right]^{\mathrm{T}}$ It's a column vector that represents the time series corresponding to the JTH source component The magnitude of the value $\boldsymbol{a}_{j}$ reflects the source component throughout the entire experiment The degree of "contribution" $\boldsymbol{s}_{j}$ at different sampling moments.

The formula (1) can also be rewritten

$\boldsymbol{x}_{i}=\sum_{j=1}^{N} a_{i j} \boldsymbol{s}_{j}, \quad i=1, \cdots, M$

2 Based on the improved convex optimization framework CTR component extraction algorithm

\subsection{Data blindness decomposition based on AVM method}

Using AVM method to blind decomposition is a major improvement point in this paper. The advantage of AVM algorithm is that the error of the fMRI model is not sensitive, which is beneficial to improve the accuracy of the blind decomposition. The above process is realized by two steps: first, the closed convex set is constructed by using the observed fMRI data. Secondly, the AVM method is used to determine the convex set endpoint and complete the blind decomposition.

\subsubsection{Structural convex set}

Using convex optimization techniques in blind decomposition, its core idea is using fMRI observation data to construct the convex sets, convex set of internal point can be obtained by linear weighted convex set endpoint, the principle of the blind the fMRI data decomposition process into the process of calculating the convex set the endpoint.

In the case of convex sets, the formula (2) shows that the fMRI data obtained from each observation can be represented as an affine combination of all source components, i.e $\boldsymbol{x}_{i} \in \operatorname{aff}\left\{\boldsymbol{s}_{1}, \cdots, s_{N}\right\}$
3 )

In this paper, we can find that the affine combination of the observed fMRI data is equal to the affine combination of the source component [14].

$\operatorname{aff}\left\{\boldsymbol{s}_{1}, \cdots, \boldsymbol{s}_{N}\right\}=\operatorname{aff}\left\{\boldsymbol{x}_{1}, \cdots, \boldsymbol{x}_{M}\right\}$

Then, the affine combination of the source component can be further expressed as its convex combination with the non-negative constraint of the source component

$$
\begin{aligned}
S & =\operatorname{conv}\left\{\boldsymbol{s}_{1}, \cdots, \boldsymbol{s}_{N}\right\}=\operatorname{aff}\left\{\boldsymbol{s}_{1}, \cdots, \boldsymbol{s}_{N}\right\} \cap R_{+}^{L} \\
& =\left\{\boldsymbol{x} \mid \boldsymbol{x}=\boldsymbol{C} \boldsymbol{\alpha}+\boldsymbol{d}, \boldsymbol{x} \geq 0, \boldsymbol{\alpha} \in R^{N-1}\right\}
\end{aligned}
$$

It can be estimated from the observed fMRI data [15].

\subsubsection{AVM based fMRI blind decomposition}

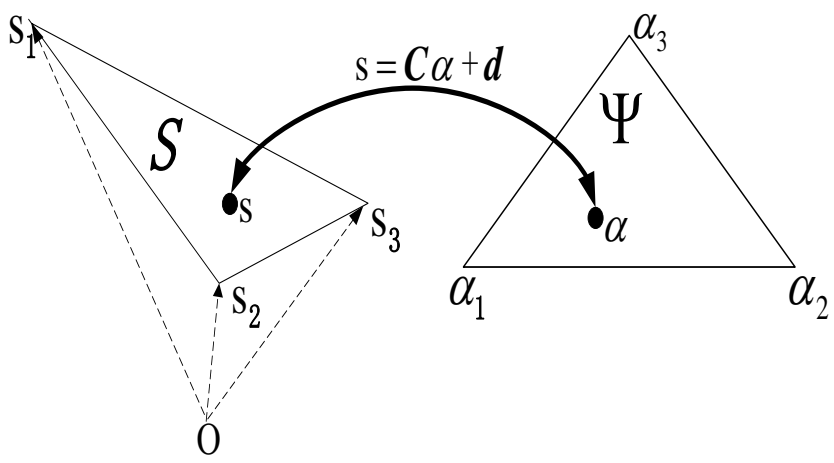

Figure 2: Conversion process of AVM $(N=3$ )

As shown in figure (2), In order to obtain the robustness of the error of the fMRI model and accurately estimate the convex set end points, the affine collection is defined using equation (5) $\Psi$ :

$$
\Psi=\left\{\boldsymbol{\alpha} \in \square^{N-1} \mid \boldsymbol{C} \boldsymbol{\alpha}+\boldsymbol{d} \succ 0\right\}
$$

In this paper, the affine collection is used $\Psi$ Maximized simplex volume $\Omega_{[13]:}$

$$
\Omega=\arg \max _{\boldsymbol{\alpha}_{1}, \cdots, \boldsymbol{\alpha}_{N} \in \Psi}\left|\operatorname{det}\left(\square\left(\boldsymbol{\alpha}_{1}, \cdots, \boldsymbol{\alpha}_{N}\right)\right)\right|
$$

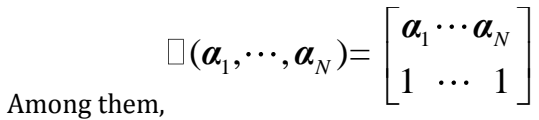

Det I want to take the determinant.The optimal solution for equation (7) is the estimated endpoint.In the end, the estimated source component can be obtained by using it.

\subsection{Analysis of somatoelement selection based on CTR component}

After blind decomposition of the observed fMRI data, the components of each decomposition can be regarded as an estimate of the true source component, which is called the estimated component [16]. The size of element values in the estimated component reflects the degree of activation of different locus. In activated voxel selection, the first of each voxel values of normalized (minus the mean, divided by the variance), and then will be the biggest voxel values before the element (article) individual selection for the estimated amount of activated voxel.

After determining the activator element of each estimator, the average time sequence of the activated body element can be obtained. Then, the estimated components corresponding to the average time sequence of the activator elements that are most relevant to the task function are selected as the CTR components. The selection process of the CTR component can be expressed as follows: 
$\hat{j}=\max _{j} \frac{E\left\{\boldsymbol{T}_{j}\right\}^{\mathrm{T}} \boldsymbol{f}}{\left\|E\left\{\boldsymbol{T}_{j}\right\}\right\| \boldsymbol{f} \|}$

$$
f(t)=r(t) \otimes h(t)
$$

\section{EXPERIMENTAL RESULTS AND ANALYSIS}

\section{1}

\section{Data description}

The experimental data were derived from the fMRI data of the ICA algorithm in BrainVoyage software, which mainly collects the Visual Structure from Motion Stimuli (VSFMS). VSFMS data is collected in an fMRI experiment of visual stimuli [17].The visual stimuli of this experiment include: moving face images, moving images, stationary face images, stationary arbitrary figures, etc. Each stimulus material appeared twice in one experiment. In the course of the experiment, the subjects were asked to keep an eye on the images that appeared, and to make a subjective judgment, whether it was a face image or a non-face image.

\section{2}

\section{Analysis of experimental results}
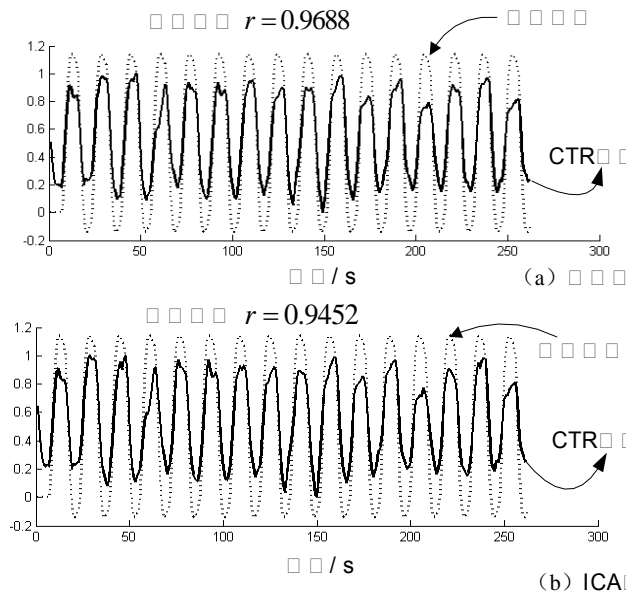

Figure 3: Component of activated voxel average time series comparison chart of CTR

In the selection of body element, ICA methods and new methods selected the largest 100 individual elements in the CTR component chosen as activator. As shown in figure 3, the proposed method selected CTR component of activated voxel with task function more relevant (ICA method corresponding to the correlation coefficient is, the proposed method corresponding to the correlation coefficient for), show that the new method of selected activated voxel more accord with the experimental stimulation results in time domain.

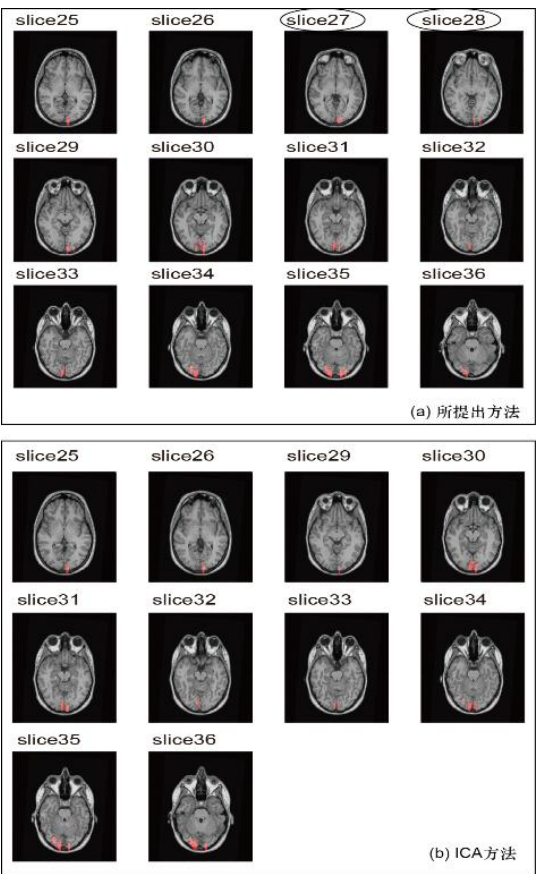

Figure 4: Distribution of space activated voxel
In terms of the spatial distribution of activator, as shown in figure 4, most ICA methods select the active body element to be selected by the proposed new method. However, the new method found some activator elements (such as slice 27, slice 28) that the ICA method did not find.

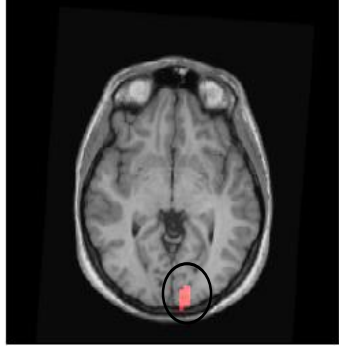

(a) Slice 27

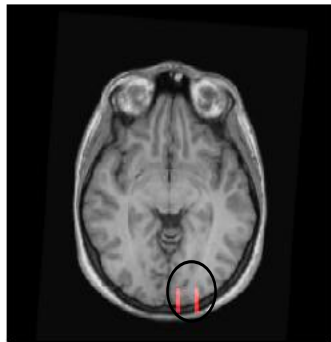

(b) Slice 28
Figure 5: Distribution of space activated voxel of slice27 and slice 28

In order to verify the effectiveness of the activator in slice 27 and slice 28 we further analyzed the activator of slice 27 and slice 28 from the two aspects of spatial distribution and time domain variation.As shown in figure 5, slice 27 and slice 28 activated voxel V1 (circle) is located in the vision, because the V1 area is mainly responsible for the brain associated with visual motion function, so the slice 27 and slice 28 activating element related to the experiment tasks.
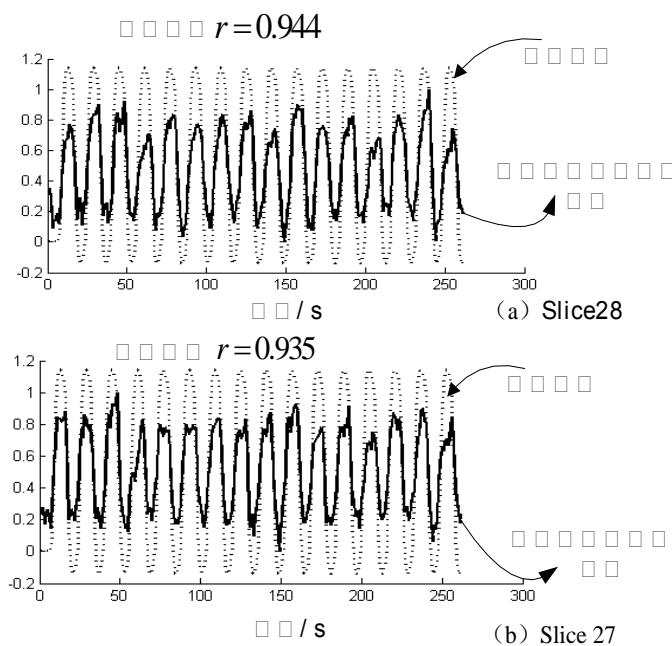

Figure 6: Activated voxel average time sequence comparison chart of slice27 and slice 28

Figure 6 shows the average slice 27 and slice 28 activated voxel time series can be found that the average time of activated voxel sequence has strong correlation with task function, and further confirmed these voxel is from the time domain by experiment task to stimulate and activate. However, these bodies have not been selected by ICA methods, and this conclusion confirms the effectiveness of the proposed approach.

\section{CONCLUSION}

Based on the characteristics of fMRI data, this paper presents a new datadriven method to blind decomposition of fMRI data and presents the results of localization analysis based on CTR component. With the help of the source component nonnegative and sparse hypothesis, by building a new method based on the observation of the fMRI data convex set, to convert the estimation problem of the source component to build on convex set and the endpoint. In convex set in the process of the endpoint is determined, using the method of equivalent maximum volume to effectively approach constructed convex set, in order to improve the accuracy of determining convex set endpoint, enhancement algorithm robust ability of fMRI model error. The experimental results show that the activated body element found by the proposed method is more consistent with the brain response to task function stimulation during the experiment. Reflect the superiority of the new methods used the more accord with fMRI data from its natural characteristics of mathematical assumptions, at the same time in the optimization process give full consideration to the effects of model error, improve the positioning accuracy of fMRI brain activation area. 


\section{ACKNOWLEDGEMENTS}

The authors gratefully acknowledge financial support from the Nature Science Fund of Guangxi Province (2016GXNSFBA380160); Key Fund Program of Guangxi Province of Department of Education(KY2015ZD143); Fund Program of Guangxi Province of Department of Education(KY2016YB193)

\section{ABOUT THE AUTHORS}

Liu Shengqian(1984-),male, Shishou in Hubei province, lecturer, gradate for Phd, main direction of studying: advanced detecting and sensing technology; Feng bao(1986-), male, Taiyuan in Shanxi Province, associate professor, Phd, main direction of studying: mode recognition, brain signal analysis, convex analysis and optimization; Zhang Lieping(1971-), male, Nankang in Jiangxi Province, professor, main direction of studying: artificial intelligence; Sun xuan(1970-), male , Songzi in Hubei province, associate professor, Phd, main direction of studying: embedded systems and applications.

\section{REFERENCES}

[1] Scantlebury, J., Bouffet, N., Laughlin E.S. 2016. White matter and information processing speed following treatment with cranial-spinal radiation for pediatric brain tumor. Neuropsychology, 30 (4), 425.

[2] Yi, J.Z., Chenglin, L., Ti-niu, T. 2016. The review and prospect of the research on brain intelligence. Journal of computer science, 39 (1), 212222 .

[3] Yang, J.L., Weiming, Z., Ni, W. 2016. Based on the fMRI brain functional connectivity detection method based on wavelet domain. Computer system application, 3 (1), 214-218.

[4] Yandong, D.L., Tan, Z.Z., Devan, H., Lirong, Y., Changlian, T., Daxing, W., Shuqiao, Y. 2004. A new method of comprehensive analysis of brain fMRI data. Chinese science: E, 34 (1), 1139-1147.

[5] Feng, J.B., Yu, Z.L., Gu, Z. 2015. Analysis of fMRI data based on sparsity of source components in signal dictionary. Neurocomputing, 156 (24), 86-95.
[6] Daubechies, J.I., Roussos, E., Takerkart, S. 2009. Independent component analysis for brain fMRI does not select for independence. Proceedings of the National Academy of Sciences, 106 (26), 10415-10422.

[7] Baofeng, J.T., Yuanyuan, Z., Yue, W. 2015. Study on the method of total wave nuclear magnetic resonance signal noise filtering based on independent component analysis, 64 (22), 229301-229301.

[8] McKeown, M. J., Sejnowski, T. J. 1998. Independent component analysis of fMRI data: examining the assumptions. Hum. Brain Mapp, 6 (56), 368-372.

[9] Li, Y., Namburi, P., Yu, Z., Guan, C., Feng, J., Gu, Z. 2009. Voxel selection in fMRI data analysis based on sparse representation. IEEE Xplore: IEEE Transactions on Biomedical Engineering, 56 (11), 2439 2451, 2009.

[10] Yamashita, O., Sato, M., Yoshioka, T., Tong, F., Kamitani, Y. 2008. Sparse estimation automatically selects voxels relevant for the decoding of fMRI activity patterns. NeuroImage, 42 (2), 1414-1429.

[11] McKeown, M. J., Makeig, S., Brown, G. G., Jung, T. P., Kindermann, S. S., Bell, A. J., Sejnowski, T. J. 1998. Analysis of fMRI data by blind separation into independent spatial components. Human Brain Mapping, 6 (2), 160 188.

[12] Depth. 1992. Magnetic resonance principle. High education press.

[13] Ma, W.K., Chan, T.H., Chi, C.Y., Wang, Y. 2010. Convex analysis for non-negative blind source separation with application in imaging in Chapter 7, Convex Optimization in Signal Processing and Communications, Editors: D. P. Palomar and Y. C. Eldar, UK: Cambridge University Press.

[14] Boyd, S. P., Vandenberghe, L. 2004. Convex Optimization. Cambridge university press.

[15] Feng, B., Yu, Z.L., Gu, Z. 2012. Identifying activated brain regions from fMRI data based on blind non-negative component separation technique[C]. Proceedings of 2012 IEEE-EMBS International Conference on Biomedical and Health Informatics. IEEE, 313-316.

[16] Kriegeskorte, N., Sorger, B., Naumer, M., Schwarzbach, J., Van Den Boogert, E., Hussy, W., Goebel, R. 2003. Human cortical object recognition from a visual motion flowfield. Journal of Neuroscience, 23 (2), 14511463. 